\title{
On the asymptotic mean value property for planar $p$-harmonic functions
}

\author{
ANGEL ARROYO and JOSÉ G. LLORENTE \\ Departament de Matemàtiques \\ Universitat Autònoma de Barcelona \\ 08193 Bellaterra. Barcelona \\ SPAIN \\ arroyo@mat.uab.cat \\ jgllorente@mat.uab.cat
}

\begin{abstract}
We show that $p$-harmonic functions in the plane satisfy a nonlinear asymptotic mean value property for $p>1$. This extends previous results of Manfredi and Lindqvist for certain range of $p$ 's.
\end{abstract}

\section{Introduction}

It is well known that harmonic functions in euclidean domains are those continous functions satisfying the usual mean value property. Actually, harmonic functions can be characterized by the so called asymptotic mean value property:

$$
u(x)=f_{B(x, r)} u(y) d y+o\left(r^{2}\right)
$$

as $r \rightarrow 0$.

It is a challenging problem to try to find similar characterizations for solutions of other nonlinear differential operators such as the $p$-laplacian. We recall that a function $u \in W_{l o c}^{1, p}(\Omega)$ is $p$-harmonic in a domain $\Omega \subset \mathbb{R}^{d}$ if it is a weak solution of the $p$-laplace equation

$$
\operatorname{div}\left(|\nabla u|^{p-2} \nabla u\right)=0
$$

Key words: mean value property, p-harmonic function. MSC2010: 31C05, 35B60, 31C45. Partially supported by grants MTM2011-24606, MTM2014-51824-P and 2014 SGR 75 . 
If $u \in C^{2}$ and $\nabla u(x) \neq 0$ then direct computation shows that

$$
\triangle_{p} u \equiv \operatorname{div}\left(|\nabla u|^{p-2} \nabla u\right)=|\nabla u|^{p-2}\left[(p-2) \frac{\triangle_{\infty} u}{|\nabla u|^{2}}+\triangle u\right]
$$

where $\triangle_{\infty}$ is the so called infinity laplacian which, for $C^{2}$ functions, is given by

$$
\triangle_{\infty} u=\sum_{i, j=1}^{d} u_{x_{i}, x_{j}} u_{x_{i}} u_{x_{j}}
$$

On the other hand it follows essentially from Taylor's formula that

$$
\begin{aligned}
\lim _{r \rightarrow 0} \frac{1}{r^{2}}\left[\frac{1}{2}\left(\sup _{B(x, r)} u+\inf _{B(x, r)} u\right)-u(x)\right] & =\frac{\triangle_{\infty} u(x)}{2|\nabla u(x)|^{2}} \\
\lim _{r \rightarrow 0} \frac{1}{r^{2}}\left(f_{B(x, r)} u-u(x)\right) & =\frac{\triangle u(x)}{2(d+2)}
\end{aligned}
$$

where $B(x, r)$ denotes the open ball centered at $x$ of radius $r$. From (1.1),(1.2) and (1.3) it can be shown that if $u \in C^{2}, \triangle_{p} u=0$ and $\nabla u(x) \neq 0$ then

$$
u(x)=\frac{p-2}{p+d} \cdot \frac{1}{2}\left(\sup _{B(x, r)} u+\inf _{B(x, r)} u\right)+\frac{2+d}{p+d} f_{B(x, r)} u(y) d y+o\left(r^{2}\right)
$$

as $r \rightarrow 0$. Since $p$-harmonic functions are $C^{1, \alpha}$ for some $0<\alpha<1$ but not $C^{2}$ in general ([U], $[\mathrm{L}])$, it is not clear whether (1.4) should be true in the general case. However, in [PMR] the authors proved that if $u \in C(\Omega) \cap W_{l o c}^{1, p}(\Omega)$ then $u$ is $p$-harmonic in $\Omega$ if and only if (1.4) holds in a weak(viscosity) sense. From [JLM] and [PMR], it follows that if $u$ is continous and satisfies (1.4) in classical sense then $u$ is $p$-harmonic.

More information is available when $d=2$. If $u$ is $p$-harmonic in a planar domain then the complex gradient $\partial u=\frac{1}{2}\left(u_{x}-i u_{y}\right)$ is a quasiregular mapping( so the critical points are isolated, unless $u$ is constant) and $u$ is $C^{\infty}$ in $\{\nabla u \neq 0\}$ (see [BI], [IM]). Lindqvist and Manfredi have recently proven that in the plane $p$-harmonicity is equivalent to the asymptotic mean value property( in classical sense) for a certain range of $p$ 's. Hereafter we denote by $D(x, r)$ the open disc of center $x$ and radius $r$.

Theorem. ([LM]) Let $\Omega \subset \mathbb{R}^{2}$ be a domain and let $1<p<p_{0}=9.52 \ldots$. Then $u \in C(\Omega) \cap W_{l o c}^{1, p}(\Omega)$ is $p$-harmonic in $\Omega$ if and only if the asymptotic expansion

$$
u(x)=\frac{p-2}{p+2} \cdot \frac{1}{2}\left(\sup _{D(x, r)} u+\inf _{D(x, r)} u\right)+\frac{4}{p+2} f_{D(x, r)} u(y) d y+o\left(r^{2}\right)
$$

holds at each $x \in \Omega$, as $r \rightarrow 0$. 
Our main result is an extension of Lindqvist and Manfredi's theorem to the whole range of $p$ 's.

Theorem 1. Let $\Omega \subset \mathbb{R}^{2}$ be a domain and let $1<p<\infty$. Then $u \in$ $C(\Omega) \cap W_{\text {loc }}^{1, p}(\Omega)$ is p-harmonic in $\Omega$ if and only if the asymptotic expansion (1.5) holds at each $x \in \Omega$, as $r \rightarrow 0$.

It is enough to prove that a $p$-harmonic function satisfies (1.5) at a critical point. Indeed, by the previous comments, continous functions satisfying (1.5) are $p$-harmonic and, on the other hand, $p$-harmonic functions satisfy

(1.5) at noncritical points. Therefore we will focus on the local behavior of a $p$-harmonic function around a critical point of multiplicity $n$. As in [LM], the method exploits the power series expansion of the complex gradient in the hodographic plane that was obtained in [IM].

\section{The hodographic representation of $u$}

Let $u$ be a $p$-harmonic function with a critical point of multiplicity $n$ at the origin and let

$$
\partial u(z)=\frac{1}{2}\left(u_{x}-i u_{y}\right)
$$

be the complex gradient of $u$. We can represent $\partial u(z)=(\chi(z))^{n}$ where $\chi$ is quasiconformal in a neighborhood of the origin and $\chi(0)=0$ (see [BI]). In the hodographic plane, $\xi=\chi(z)$, and, according to [IM], the inverse of $\chi$ is given by

$$
z=H(\xi)=\sum_{k=n+1}^{\infty}\left[A_{k}\left(\frac{\xi}{|\xi|}\right)^{k}+\varepsilon_{k} \overline{A_{k}}\left(\frac{\bar{\xi}}{|\xi|}\right)^{k}\right]\left(\frac{\xi}{|\xi|}\right)^{-n}|\xi|^{\lambda_{k}}
$$

where $A_{k} \in \mathbb{C}, A_{n+1} \neq 0$ and

$$
\sum_{k=n+1}^{\infty} k\left|A_{k}\right|^{2}<\infty
$$

Furthermore,

$$
\varepsilon_{k}=\frac{\lambda_{k}+n-k}{\lambda_{k}+n+k}, \quad \lambda_{k}=\frac{1}{2}\left(\sqrt{4 k^{2}(p-1)+n^{2}(p-2)^{2}}-n p\right) .
$$

From (2.3) it is easy to check that

$$
0<\lambda_{k}<\frac{k^{2}-n^{2}}{n}, \quad\left|\varepsilon_{k}\right|<\frac{k-n}{k+n}
$$

Equation (2.1) can be interpreted as the "hodographic representation" of the point $z=x+i y$ near the origin. 
Therefore, if $\xi=r e^{i \theta}$, we can rewrite (2.1) as

$$
H\left(r e^{i \theta}\right)=e^{-i n \theta} \sum_{k=n+1}^{\infty} r^{\lambda_{k}} \varphi_{k}(\theta)
$$

where

$$
\varphi_{k}(\theta)=A_{k} e^{i k \theta}+\varepsilon_{k} \overline{A_{k}} e^{-i k \theta}
$$

for each $k=n+1, n+2, \ldots$ We can split $H(\xi)$ into its real and imaginary parts, i.e., $H(\xi)=\tilde{z}(\xi)=\tilde{x}(\xi)+i \tilde{y}(\xi)$.

Lets denote by $\tilde{u}$ the hodographic representation of $u$, i.e.,

$$
\tilde{u}(\xi)=(u \circ H)(\xi) .
$$

Moreover, we can easily write $\partial u(z)$ in terms of $\xi$ :

$$
\partial u(z)=\partial u(H(\xi))=\xi^{n}
$$

or, equivalently, if $\xi=r e^{i \theta}$,

$$
\left\{\begin{array}{l}
u_{x}=2 r^{n} \cos (n \theta) \\
u_{y}=-2 r^{n} \sin (n \theta)
\end{array}\right.
$$

Proposition 2.1. Let $u$ be a p-harmonic function with a critical point of order $n$ at $z=0$. Then the hodographic representation $\tilde{u}$ has the following power series expansion in a neighborhood of $\xi=0$ :

$$
\tilde{u}(\xi)=u(0)+4 \sum_{k=n+1}^{\infty} \mu_{k}|\xi|^{n+\lambda_{k}} \mathfrak{R e}\left\{A_{k}\left(\frac{\xi}{|\xi|}\right)^{k}\right\},
$$

where $\mu_{k}=\frac{\lambda_{k}}{\lambda_{k}+n+k}$. Moreover, $0 \leq \mu_{k}<1-\frac{n}{k}$.

Proof. Using (2.7), we compute $\tilde{u}_{r}$ :

$$
\tilde{u}_{r}=(u \circ H)_{r}=u_{x} \tilde{x}_{r}+u_{y} \tilde{y}_{r}=2 r^{n}\left[\tilde{x}_{r} \cos (n \theta)-\tilde{y}_{r} \sin (n \theta)\right]
$$

the expression in brackets being equal to $\mathfrak{R e}\left\{e^{i n \theta} H_{r}\right\}$. Replacing (2.5) in the previous equation we get

$$
\tilde{u}_{r}=2 r^{n} \mathfrak{R e}\left\{e^{i n \theta} H_{r}\right\}=4 \sum_{k=n+1}^{\infty} \lambda_{k}\left(1+\varepsilon_{k}\right) r^{n+\lambda_{k}-1} \mathfrak{R e}\left\{A_{k} e^{i k \theta}\right\} .
$$

Integrate with respect to $r$ to complete the proof. The bound on $\mu_{k}$ follows from (2.4).

Remark. From now on, we can assume without loss of generality that $u(0)=0$. 


\section{Quantitative injectivity estimates for the first term of $H$}

We define the mapping $\mathcal{A}(\xi)$ as the first term in the power series expansion of $H$ :

$$
\mathcal{A}(\xi)=\left[A_{n+1}\left(\frac{\xi}{|\xi|}\right)^{n+1}+\varepsilon_{n+1} \overline{A_{n+1}}\left(\frac{\bar{\xi}}{|\xi|}\right)^{n+1}\right]\left(\frac{\xi}{|\xi|}\right)^{-n}|\xi|^{\lambda_{n+1}}
$$

We define $\widetilde{\mathfrak{U}}(\xi)$ to be the first term in the power series expansion of $\tilde{u}$,

$$
\widetilde{\mathfrak{U}}(\xi)=4 \mu_{n+1}|\xi|^{n+\lambda_{n+1}} \mathfrak{R e}\left\{A_{n+1}\left(\frac{\xi}{|\xi|}\right)^{n+1}\right\} .
$$

For simplicity, we will use hereafter the notations $a \lesssim b($ resp. $a \approx b$ ) to indicate that $a \leq C b$ (resp. $C^{-1} a \leq b \leq C a$ ) for some positive constant $C$ independent of $a$ and $b$.

Lemma 3.1. The following estimates hold in a neighborhood of $\xi=0$ :

$$
\begin{aligned}
|\widetilde{u}(\xi)-\widetilde{\mathfrak{U}}(\xi)| & \lesssim|\xi|^{n+\lambda_{n+2}} \\
|H(\xi)-\mathcal{A}(\xi)| & \lesssim|\xi|^{\lambda_{n+2}} \\
|\mathcal{A}(\xi)| \approx|H(\xi)| & \approx|\xi|^{\lambda_{n+1}}
\end{aligned}
$$

Proof. From (2.2), ( 2.4) and the fact that $0 \leq \mu_{k}<1$ we get in particular that the sequence $\left(A_{k}\right)$ is bounded and that $\left|\epsilon_{k}\right|<1$ for all $k$. Since $\left(\lambda_{k}\right)$ is increasing, (3.3), (3.4) and (3.5) follow from the estimate

$$
\sum_{k=n+2}^{\infty}|\xi|^{\lambda_{k}}=O\left(|\xi|^{\lambda_{n+2}}\right)
$$

Now an elementary computation shows that there is $C=C(p)>0$ such that $\lambda_{k}-\lambda_{n+2} \geq C(k-(n+2))$ for all $k \geq n+2$.This implies (3.6) and proves the lemma.

Now, we study the behavior of $\mathcal{A}$ and we give an injectivity estimate. For this purpose, we will need the help of the following elementary lemma whose proof is omitted.

Lemma 3.2. Let $\rho>0, \lambda>0$ and $t \in \mathbb{R}$. Then for all $k \in \mathbb{N}$,

$$
\left|\rho e^{i k t}-1\right| \leq k\left|\rho e^{i t}-1\right| .
$$

Furthermore, if $\Lambda>1$ and if $\Lambda^{-1} \leq \rho \leq \Lambda$ then there is a constant $C=$ $C(\lambda, \Lambda)>0$ such that

$$
\left|\rho^{\lambda} e^{i t}-1\right| \geq C \rho^{\lambda-1}\left|\rho e^{i t}-1\right| .
$$


Lemma 3.3. The mapping $\mathcal{A}: \mathbb{C} \rightarrow \mathbb{C}$ is bijective and satisfies

$$
|\mathcal{A}(\xi)-\mathcal{A}(\zeta)| \geq\left. C|| \xi\right|^{\lambda_{n+1}-1} \xi-|\zeta|^{\lambda_{n+1}-1} \zeta \mid
$$

where $C=\left(1-(2 n+1)\left|\varepsilon_{n+1}\right|\right)\left|A_{n+1}\right|$.

Proof. First, we observe that from (2.4) for $k=n+1$ we obtain that $0<$ $\lambda_{n+1}<2+\frac{1}{n}$ and that

$$
\left|\varepsilon_{n+1}\right|<\frac{1}{2 n+1}
$$

We show first that $\mathcal{A}$ is surjective. We write $\lambda \equiv \lambda_{n+1}, \epsilon \equiv \epsilon_{n+1}$ and $A \equiv A_{n+1}$. Then

$$
\mathcal{A}\left(r e^{i \theta}\right)=r^{\lambda} e^{i \theta}\left(A+\epsilon \bar{A} e^{-i 2(n+1) \theta}\right)
$$

Assume, for simplicity, that $A=1$. Then we can write

$$
\mathcal{A}\left(r e^{i \theta}\right)=r^{\lambda}\left|1+\epsilon e^{-i 2(n+1) \theta}\right| e^{i f(\theta)}
$$

where $f(\theta)=\theta+\arg \left(1+\epsilon e^{-i 2(n+1) \theta}\right)$ and

$$
m(\theta)=\left|1+\epsilon e^{-i 2(n+1) \theta}\right|=\sqrt{1+\varepsilon^{2}+2 \varepsilon \cos (2(n+1) \theta)} .
$$

To prove that $\mathcal{A}$ is surjective, let $w=s e^{i t} \in \mathbb{C}$ such that $w \neq 0$ (if $w=0$ it is obvious that $\mathcal{A}(0)=0)$. Since $f(0)=0$ and $f(2 \pi)=2 \pi$, by continuity we can pick $k \in \mathbb{Z}$ and $\theta \in[0,2 \pi]$ such that $t+2 k \pi \in[0,2 \pi]$ and $f(\theta)=t+2 k \pi$. Then $e^{i f(\theta)}=e^{i t}$. For that $\theta$, choose $r>0$ so that

$$
r^{\lambda} m(\theta)=s
$$

Then we have shown that $\mathcal{A}\left(r e^{i \theta}\right)=w$ so the surjectiveness of $\mathcal{A}$ follows.

To finish the proof of the lemma, it is enough to prove (3.9), which is a quantitative form of injectiveness. By (3.1),

$$
\begin{aligned}
|\mathcal{A}(\xi)-\mathcal{A}(\zeta)| & \geq\left.\left|A_{n+1}\right||| \xi\right|^{\lambda} \frac{\xi}{|\xi|}-|\zeta|^{\lambda} \frac{\zeta}{|\zeta|} \mid- \\
& -\left.\left|A_{n+1}\right||\varepsilon||| \xi\right|^{\lambda}\left(\frac{\bar{\xi}}{|\xi|}\right)^{2 n+1}-|\zeta|^{\lambda}\left(\frac{\bar{\zeta}}{|\zeta|}\right)^{2 n+1} \mid
\end{aligned}
$$

Now apply (3.7) with $\rho=\left|\frac{\xi}{\zeta}\right|^{\lambda}, e^{i t}=\frac{\xi / \zeta}{|\xi / \zeta|}$ and $k=2 n+1$, and multiply both sides of the inequality by $|\zeta|^{\lambda}$. Then

$$
\left.|| \xi\right|^{\lambda}\left(\frac{\xi}{|\xi|}\right)^{2 n+1}-|\zeta|^{\lambda}\left(\frac{\zeta}{|\zeta|}\right)^{2 n+1}|\leq(2 n+1)||\xi|^{\lambda} \frac{\xi}{|\xi|}-|\zeta|^{\lambda} \frac{\zeta}{|\zeta|} \mid .
$$


Replacing this expression in (3.12) we obtain

$$
|\mathcal{A}(\xi)-\mathcal{A}(\zeta)| \geq\left.\left|A_{n+1}\right|(1-(2 n+1)|\varepsilon|)|| \xi\right|^{\lambda} \frac{\xi}{|\xi|}-|\zeta|^{\lambda} \frac{\zeta}{|\zeta|} \mid .
$$

so the proof is finished.

Lemma 3.4. Let $\Lambda>1$. Then there is a constant $C=C\left(n, p, \Lambda,\left|A_{n+1}\right|\right)>$ 0 such that for any $\xi, \zeta \in \mathbb{C}$ with $\Lambda^{-1}|\zeta| \leq|\xi| \leq \Lambda|\zeta|$ we have

$$
|\mathcal{A}(\xi)-\mathcal{A}(\zeta)| \geq C|\xi|^{\lambda_{n+1}-1}|\xi-\zeta| \text {. }
$$

Proof. Apply (3.8) with $\rho=\left|\frac{\xi}{\zeta}\right|$ and $e^{i t}=\frac{\xi / \zeta}{|\xi / \zeta|}$ and multilply by $|\zeta|^{\lambda}$ to obtain

$$
\left.|| \xi\right|^{\lambda} \frac{\xi}{|\xi|}-\left.|\zeta|^{\lambda} \frac{\zeta}{|\zeta|}|\geq C| \xi\right|^{\lambda-1}|\xi-\zeta|
$$

Then the lemma follows from (3.9) together with (3.14).

\section{The perturbation method}

Given $\xi$ in the hodographic plane, set $z=H(\xi), \zeta=\mathcal{A}^{-1}(z)$ and $w=H(\zeta)$. Then

$$
\xi=\chi(z), \quad \zeta=\chi(w)=\mathcal{A}^{-1}(H(\xi))
$$

Since $|z|=|\mathcal{A}(\zeta)| \approx|H(\zeta)|=|w|$ by (3.5) it follows from quasiconformality $([\mathrm{A}])$ that $|\xi|=|\chi(z)| \approx|\chi(w)|=|\zeta|$. We recall that $u(z)=(\tilde{u} \circ \chi)(z)$ and that $\widetilde{\mathfrak{U}}$ is given by (3.2). Following [LM], define the functions

$$
\mathfrak{U}(z)=\left(\widetilde{\mathfrak{U}} \circ \mathcal{A}^{-1}\right)(z)
$$

Lemma 4.1. Let $\Lambda>1$. There is a constant $C=C\left(n, p, \Lambda,\left|A_{n+1}\right|\right)>0$ such that for any $\xi, \zeta \in \mathbb{C}$ with $\Lambda^{-1}|\zeta| \leq|\xi| \leq \Lambda|\zeta|$ then

$$
|\widetilde{\mathfrak{U}}(\xi)-\widetilde{\mathfrak{U}}(\zeta)| \leq C|\xi|^{n}|\mathcal{A}(\xi)-\mathcal{A}(\zeta)| .
$$

Proof. From (3.2), the fact that $0 \leq \mu_{k}<1$ if $k \geq n$ and direct computation it follows that

$$
|\widetilde{\mathfrak{U}}(\xi)-\widetilde{\mathfrak{U}}(\zeta)| \leq C\left|A_{n+1}\right||\xi|^{n+\lambda_{n+1}-1}|\xi-\zeta|
$$

where $C=C(n, \Lambda)>0$. Then the conclusion follows from Lemma 3.4. 
Corollary 4.1. Let $\xi$ and $\zeta$ be as in the beginning of the section. Then the following estimate holds in a neighborhood of $\xi=0$ :

$$
|\widetilde{\mathfrak{U}}(\xi)-\widetilde{\mathfrak{U}}(\zeta)| \lesssim|\xi|^{n+\lambda_{n+2}}
$$

Proof. Use the fact that $|\xi| \approx|\zeta|$, Lemma 4.1 and estimate (3.4).

Now we are ready to prove the following singular expansion of a $p$ harmonic function.

Proposition 4.1. Let $u$ be a p-harmonic function with a singularity of order $n$ at $z=0$ and $u(0)=0$. Then $u$ can be written as

$$
u(z)=\mathfrak{U}(z)+O\left(|z|^{\frac{n+\lambda_{n+2}}{\lambda_{n+1}}}\right)
$$

in a neighborhood of $z=0$.

Proof. By (2.6) and (4.1) we can write

$$
u(z)-\mathfrak{U}(z)=\tilde{u}(\xi)-\widetilde{\mathfrak{U}}(\zeta)=\widetilde{\mathfrak{U}}(\xi)-\widetilde{\mathfrak{U}}(\zeta)+\tilde{u}(\xi)-\widetilde{\mathfrak{U}}(\xi) .
$$

By (3.3), (3.5) and Corollary 4.1 we get

$$
|u(z)-\mathfrak{U}(z)| \lesssim|\xi|^{n+\lambda_{n+2}} \approx|H(\xi)|^{\frac{n+\lambda_{n+2}}{\lambda_{n+1}}}=|z|^{\frac{n+\lambda_{n+2}}{\lambda_{n}+1}}
$$

so the proof is finished.

\section{$5 \quad$ Proof of Theorem 1}

As before, we will write $\lambda$ and $\varepsilon$ instead of $\lambda_{n+1}$ and $\varepsilon_{n+1}$, respectively. We can assume without loss of generality that $A_{n+1}=1$. Then

$$
\mathcal{A}\left(r e^{i \theta}\right)=r^{\lambda} e^{-i n \theta}\left(e^{i(n+1) \theta}+\varepsilon e^{-i(n+1) \theta}\right)
$$

and $\left|\mathcal{A}\left(r e^{i \theta}\right)\right|=r^{\lambda} m(\theta)$, where $m(\theta)$ is given by (3.11). Furthermore

$$
\widetilde{\mathfrak{U}}\left(r e^{i \theta}\right)=4 \mu r^{n+\lambda} \cos ((n+1) \theta) .
$$

where $\mu=\mu_{n+1}$.

Denote by $D_{R}=D(0, R)$ the open disc centered at 0 with radius $R>0$ and define the "hodographic disc" $\widetilde{D_{R}}$ as $\mathcal{A}^{-1}\left(D_{R}\right)$. Then, a point $r e^{i \theta}$ of the hodographic plane belongs to $\widetilde{D_{R}}$ if and only if $\left|\mathcal{A}\left(r e^{i \theta}\right)\right|<R$. Then, $\widetilde{D_{R}}$ can be described, in polar coordinates, as

$$
\widetilde{D_{R}}=\left\{r e^{i \theta}: r<\left(\frac{R}{m(\theta)}\right)^{1 / \lambda}\right\}
$$


Now we define the function $J(\zeta)$ as the absolute value of the jacobian of $\mathcal{A}(\zeta)$. Computing $J(\zeta)$ in polar coordinates we get

$$
J\left(r e^{i \theta}\right)=\lambda r^{2(\lambda-1)}\left(1-(2 n+1) \varepsilon^{2}-2 n \varepsilon \cos (2(n+1) \theta)\right),
$$

(Observe that, since $|\epsilon|<(2 n+1)^{-1}$, the expression in the right hand side of (5.1) is positive).

Lemma 5.1. The p-harmonic function $\mathfrak{U}(z)$ given by (4.1) satisfies the following properties, for small enough $R>0$ :

$$
\begin{gathered}
\sup _{D_{R}} \mathfrak{U}+\inf _{D_{R}} \mathfrak{U}=0, \\
\int_{D_{R}} \mathfrak{U}=0 .
\end{gathered}
$$

Proof. By (4.1), we need to study the behavior of $\widetilde{\mathfrak{U}}(\xi)$ in $\widetilde{D_{R}}$. Then, (5.2) is a direct consequence of the symmetries of $\widetilde{D_{R}}$. To show (5.3), observe that, by change of variables

$$
\int_{D_{R}} \mathfrak{U}(z) d z=\int_{\widetilde{D_{R}}} \widetilde{\mathfrak{U}}(\zeta) J(\zeta) d \zeta
$$

and using polar coordinates in (5.4) we get

$$
\int_{D_{R}} \mathfrak{U}(z) d z=4 \mu \lambda \int_{0}^{2 \pi} \int_{0}^{r(\theta)} r^{n+3 \lambda-1} \cos ((n+1) \theta) j(\theta) d r d \theta
$$

where

$$
r(\theta)=\left(\frac{R}{m(\theta)}\right)^{1 / \lambda}, \quad j(\theta)=1-(2 n+1) \epsilon^{2}-2 n \epsilon \cos (2(n+1) \theta)
$$

and $m(\theta)$ is given by (3.11). Now (5.3) follows directly from (5.5) and the symmetry properties of $m(\theta)$ and $j(\theta)$.

Lemma 5.2. The inequality

$$
\frac{n+\lambda_{n+2}}{\lambda_{n+1}}>2
$$

holds for each $1<p<\infty$ and each $n \geq 1$.

Proof. From (2.3) and some computation it follows that inequality (5.6) is equivalent to

$$
n(p+2) \sqrt{n^{2} p^{2}+16(n+1)(p-1)}>n^{2} p^{2}+\left(-2 n^{2}+8 n\right) p-\left(2 n^{2}+8 n\right) .
$$


Now we distinguish two cases. If $n=1$ then (5.7) becomes

$$
(p+2) \sqrt{p^{2}+32(p-1)}>p^{2}+6 p-10 .
$$

If the right hand is negative then the inequality follows. Otherwise, squaring the previous inequality we get

$$
2 p^{3}+7 p^{2}+10 p-19>0 \text {. }
$$

which holds for each $p>1$ since the left-hand side is increasing in $p$ and vanishes for $p=1$. This proves (5.7) when $n=1$.

Now assume $n \geq 2$ and observe that $\sqrt{n^{2} p^{2}+16(n+1)(p-1)} \geq n p$ for each $p>1$. Then (5.7) would follow if:

$$
n^{2} p(p+2)>n^{2} p^{2}+\left(-2 n^{2}+8 n\right) p-\left(2 n^{2}+8 n\right),
$$

which is equivalent to

$$
(2 n-4) p+n+4>0,
$$

and holds trivially if $n \geq 2$. This finishes the proof of the lemma.

Proof of Theorem 1. As stated at the introduction, we only need to prove that planar $p$-harmonic functions satisfy (1.5) since the converse is well known. We also discussed there that (1.5) need only to be checked at a critical point. Therefore, we can assume that $x=0, u(0)=0$ and that 0 is a critical point of $u$.

Let $r>0$ be small enough. By Proposition 4.1 and Lemma 5.1,

$$
\int_{D(0, r)} u=O\left(r^{\frac{n+\lambda_{n+2}}{\lambda_{n+1}}}\right) .
$$

and

$$
\frac{1}{2}\left(\sup _{D(0, r)} u+\inf _{D(0, r)} u\right)=O\left(r^{\frac{n+\lambda_{n+2}}{\lambda_{n+1}}}\right) .
$$

Finally, combine (5.9), (5.10) and divide by $r^{2}$ to obtain that for any $\alpha \in \mathbb{R}$

$$
\frac{1}{r^{2}}\left[\alpha\left(\frac{1}{2} \sup _{D(0, r)} u+\frac{1}{2} \inf _{D(0, r)} u\right)+(1-\alpha) f_{D(0, r)} u\right]=O\left(r^{\frac{n+\lambda_{n+2}}{\lambda_{n+1}}-2}\right) .
$$

By 5.2 the exponent of $r$ in the right-hand side is strictly positive. Therefore, taking limits as $r \rightarrow 0$, we show that (1.5) holds at the origin and we conclude the proof.

Remark. The proof actually shows that if $x$ is a critical point of the $p$ harmonic function $u$ then (1.5) still holds at $x$ if the coefficients $(p-2) /(p+2)$ and $4 /(p+2)$ are replaced by $\alpha$ and $1-\alpha$ for arbitrary $\alpha$. 


\section{References}

[A] L. V. Ahlfors, Lectures on quasiconformal mappings, Providence, 2006 .

[BI] B. Bojarski, T. IwANIEC, p-harmonic equation and quasiregular mappings, Partial Differential Equations, Banach Center Publications, 19, 1987, pp. 25-38.

[IM] T. Iwaniec, J. Manfredi, Regularity of p-harmonic functions on the plane, Revista Matemática Iberoamericana, 5, (1989), 1-19.

[JLM] P. Juutinen, P. Lindqvist, J. Manfredi, On the equivalence of viscosity solutions and weak solutions for a quasi-linear elliptic equation, SIAM, J. Math. Anal., 33, (2001), 699-717.

[L] J. LEwIS, Regularity of the derivatives of solutions to certain degenerate elliptic equations, Indiana Univ. Math. J. 32, (1983), 849-858.

[LM] P. Lindqvist, J. MAnfRedi, On the mean value property for the $p$-Laplace equation in the plane, Proceedings of the American Mathematical Society, (to appear).

[PMR] M. Parviainen, J. Manfredi, J. D. Rossi, An asymptotic mean value characterization for $p$-harmonic functions, Proceedings of the American Mathematical Society, 138, N.3, (2010), 881-889.

[U] N. N. Ural'tseva, Degenerate quasilinear elliptic systems, Zap. Naučn. Sem. 7(1968), 184-222. Otdel. Math. Inst. Steklov, Leningrad (in russian). 